\title{
GaiaNIR - A future all-sky astrometry mission
}

\author{
David Hobbs ${ }^{1}$ and Erik $\mathrm{H} \mathrm{g}^{2}$ \\ ${ }^{1}$ Lund Observatory, Box 43, 22100, Lund Sweden \\ email: david@astro.lu.se \\ ${ }^{2}$ Niels Bohr Institute, Juliane Maries Vej 30, 2100 Copenhagen $\varnothing$, Denmark \\ email: ehoeg@hotmail.dk
}

\begin{abstract}
With the launch of Gaia in December 2013, Europe entered a new era of space astrometry following in the footsteps of the very successful Hipparcos mission. A weakness of Gaia is that it only operates at optical wavelengths. However, much of the Galactic centre and the spiral arm regions are obscured by interstellar extinction. An obvious improvement on Gaia is to include the Near-Infra-Red (NIR) which requires the use of new types of detectors. Additionally, to scan the entire sky and measure global absolute parallaxes the spacecraft must have a constant rotation resulting in a moving image that must be compensated for by, for example, operating the detectors in Time Delayed Integration (TDI) mode. If these technical issues can be solved a new Gaia-like mission separated by a 20 year interval would give; 1) NIR all-sky astrometry and photometry to penetrate the obscured regions and to observe intrinsically red objects with almost diffraction limited resolution; 2) improved proper motions with fourteen times smaller errors than from Gaia alone opening up new science cases, such as long period exoplanets and accurate halo measurements; 3) allow the slowly degrading accuracy of the Gaia reference frame, which will be the basis for future astronomical measurements, to be reset.
\end{abstract}

Keywords. Astrometry, Catalogs, Reference systems, Galaxy: kinematics and dynamics

\section{Introduction}

The current Gaia mission has only just begun to revolutionize our understanding of the Galaxy. The first Gaia data release gave 5 parameter astrometry for more than 2 million sources but this gives just a hint of what is about to come with the second release. In April 2018 we expect to release 5 parameter astrometry for more than 1 billion sources and subsequent releases will give increasingly accurate and comprehensive sets of astrophysical data. Gaia will eventually provide positions, absolute parallaxes and proper motions, to unprecedented accuracies $\left(20-25 \mu \mathrm{as}\left(\mathrm{yr}^{-1}\right)\right.$ at $\left.\mathrm{G}=15\right)$, with the addition of all-sky homogeneous multi-colour photometry and spectroscopy. These unique capabilities go well beyond and are complementary to the science cases being addressed by ground based surveys (such as RAVE, SDSS, Pan-Starrs, APOGEE, LSST, etc).

The most obvious way to improve on Gaia's capabilities is to extend them to allsky absolute NIR astrometry allowing the new mission to probe through the Galactic dust to observe the structure and kinematics of the star forming regions in the disk, the spiral arms and the bulge region to give model independent distances and proper motions in these obscured parts of the sky. A new mission launched with an interval of 20 years (around 2035-2040) would allow new measurements of objects already in the Gaia catalogue to be combined with older data giving improved proper motions with fourteen times smaller errors. Parallaxes would also be improved in such joint solutions by a factor of $\sqrt{2}$ assuming the two missions are of equal duration. After the publication of the final Gaia catalogue the positions of stars will be accurately known at the chosen 
reference epoch (currently 2017) and linked to the VLBI reference frame. However, this accurate positional information will slowly degrade due to the small uncertainties in the proper motions of the stars. Hence, it is necessary to repeat the measurements of Gaia after about 20 years to maintain the positional accuracy and the optical reference frame.

The accuracy of the mission should be at least that of Gaia using tried and trusted instrumentation, techniques, and lessons learned from Gaia. To achieve these goals we need to explore the feasibility and technological developments needed to manufacture space qualified optical and NIR (400-2000 nm) TDI sensors with passive cooling. To maintain ESA's leadership in all-sky space astrometry it is highly desirable to develop such detector technology within Europe. The most promising NIR sensors today seem to be hybrid $\mathrm{HgCdTe}-\mathrm{CMOS}$ multiplexing sensors which can also support TDI mode. A more limited sensitivity from 900 to $1600 \mathrm{~nm}$ in a part of the field of view may be considered if CCDs are also used. In 2016 we successfully proposed such a technology study to ESA (Hobbs et al. (2016)) in a call for "New Science Ideas" to be investigated for technologies not yet sufficiently mature. It is hoped that these ideas may become candidates for future missions in the ESA Science Program.

\section{NIR astrometry and photometry}

Much of the Galactic centre and the spiral arm regions are obscured by interstellar extinction so a NIR mission is needed to astrometrically map these regions for the first time. Linking the individual motions of obscured objects with those from the earlier Gaia optical survey would give a much more complete picture of the dynamics of the Galaxy's interior. The complex nature of the Galaxy with its bulge, bar and spiral structure can excite stars to radially migrate and induce disk heating (Friedli et al. (1994), Sellwood and Binney (2002)) and accurate measurements of the 3-dimensional motion and properties of these obscured stars are needed to trace the dynamical history and evolution of our Galaxy. To study dark matter in the inner disk of the Galaxy we need NIR measurements, avoiding the need to use extinction maps, that may eventually resolve questions regarding the nature of dark matter by showing us whether the Galaxy has a cored or cusped dark matter halo (Governato et al. (2012)), whether there are thin, disc-like components of dark matter, and whether the spiral arms have their own dark matter components. Similarly, NIR is needed to study stars belonging to clusters which have similar age and metallicity, having formed from the same cloud of gas and dust, and can be used to probe the galactic disk structure and formation rate (Kuhn et al. (2015)).

Gaia is not good at detecting very faint red objects especially in extinction regions, examples include Red Dwarfs (RD), cool White Dwarfs (WD), Brown Dwarfs (BD) and free-floating planets. An NIR capable mission would allow such objects to be detected within a large volume but also crucially in the extinction (open clusters and star forming) regions that are of great interest and would shed light on the Initial Mass Function (IMF) of these regions. An NIR facility will allow to characterize the cool WD population much better than Gaia in the optical, which has limited capabilities (Carrasco et al. (2014)) both in terms of detection and parametrization. The study of WDs provides key information about the late stages of the star's life and comparison of the empirical and theoretical Luminosity Functions (LF) of WDs one can derive the age of the Galaxy and its star formation rate. Free-floating planets are interesting to study if they were ejected from planetary systems or formed in collapsing dust clouds in a similar manner to stars.

High-precision astrometry in the NIR will have an impact on a wide variety of stellar physics topics particularly in obscured regions in the Galactic plane and Galactic centre which are the birthplaces of most stellar clusters and associations. Gaia will greatly 
extend our current knowledge of clusters but they will mostly be located at high galactic latitudes and may not be fully representative of the conditions in the disk. The binary orbits of exotic objects, such as neutron stars and stellar-mass black holes, will benefit from improved mass estimates and may allow their equations-of-state to be accurately constrained for the first time. Extension to NIR will also enable extensive local tests of stellar standard candles and their period-luminosity relations since the key uncertainties of variable extinction and metallicity are significantly reduced at longer wavelengths. Finally, AGB stars are very bright in the NIR. Mira's are much more numerous than Cepheids and the period-luminosity relation of $\mathrm{OH} / \mathrm{IR}$ stars are not well known. GaiaNIR can better characterize these objects as standard candles improving the distance scale.

\section{Improved proper motions and parallaxes}

Gaia is a fabulous mission for detecting and characterising nearby streams that cross the disk of the Milky Way but it will not be sufficient to discover and characterise most of the stream-like structures in the halo. A new mission could be combined with the older Gaia catalogue to give a much longer baseline, with very accurate proper motions (a factor of 14 better in the two components) and improved parallaxes needed to reach larger distances. Dynamical studies in the outer halo would be greatly enhanced resolving tangential motions in streams and local dwarf galaxies, with a potential accuracy of $2-3 \mathrm{~km} \mathrm{~s}^{-1}$ for samples out to $\sim 100 \mathrm{kpc}$. This will provide great insight into the gravitational potential in the outer reaches of the Milky Way and halo streams are sensitive probes. Finding "gaps" in the streams may reveal the influence of dark matter sub-haloes in the Milky Way's halo and allow us to determine the dark matter distribution at large radii, including any flattening of the potential, and the total mass of the Galaxy.

Hyper-Velocity Stars (HVSs) originate from gravitational interactions with massive black holes. Very accurate proper motion measurements are a key tool to study these objects. When combined with radial velocities the three dimensional space velocity is obtained. Unfortunately, known HVSs are distant and on largely radial trajectories. Some HVSs originate in the Galactic centre while others have an origin in the disk but an origin in the Magellanic Clouds or beyond is also possible. Accurate proper motions are needed to reconstruct their trajectories and distinguish between the different possible origins. Gnedin et al. (2005) have shown that precise proper motions of HVSs would give significant constraints on the structure (axis ratios and orientation of triaxial models) of the Galactic halo. Adding NIR would also allow us to probe more deeply into the Galactic centre and to detect small populations of HVSs closer to their ejection location.

Astrometrically resolving internal dynamics of nearby galaxies, such as M31, dwarf spheroid galaxies, globular clusters, the Large and Small Magellanic Clouds (LMC, SMC), sets requirements on the accuracy. For example, the LMC has a parallax of $20 \mu$ as and an accuracy of about $10 \%$ is needed, which is just within the reach of Gaia. Precise mapping of dark matter (sub-)structure throughout local group and beyond is possible with accurate proper motions. Gaia will not be able to directly measure internal motions of the nearby galaxies. However, by combining Gaia with a new Gaia-like mission opens up the new tantalising possibility of measuring their internal motions and thus astrometrically resolving the dynamics within the Local Group.

A new astrometric mission would allow the detection of planets with significantly longer periods than by Gaia alone. The longest detectable period using astrometry roughly coincides with the mission lifetime. This sets a maximum period $P \sim 5$ years for the Gaia nominal mission, or $P \sim 10$ years if an extension is granted. A new mission launched 20 years after Gaia would increase the detectable period to about 30 years making Saturn 
( $P=29$ years) like planets detectable. Microlensing and imaging can detect some long period planets but only astrometry can properly characterise tens of thousands of planetary systems. This is required to fully understand planetary systems and their evolution, including the effect of migration of giant outer planets through the habitable zone and to find planetary systems like our own, assuming they exist! A similar limit holds for longperiod binary stars which have much larger astrometric signatures, it may be possible to detect them even if their period is somewhat longer than the limit above, possibly to $\sim 40$ years. Accurate astrometry over a longer time span would also benefit solar system measurements improving our understanding of asteroid families and the mechanisms that lead to the injection of asteroids on Earth-crossing trajectories.

\section{The reference frame}

The Gaia optical reference frame based on quasars will slowly degrade over time due to errors in its orientation and spin and due to small proper motion patterns which are not accounted for. Additionally, the catalogue accuracy will decay more rapidly due to errors in the measured proper motions. Dense and accurate reference grids are needed for forthcoming Extreme, Giant and Overwhelming telescopes but also for smaller instruments currently operating or being planned. The detection of the reference frame quasars solely from zero linear proper motion and parallax is possible, reducing the need for spectra. Quasars will have some apparent proper motions due to time-dependent source structure. These proper motions are probably not linear and this can be used to distinguish them.

An important aspect of reference frames is to link them, cross-matching with absolute coordinates, to reference frames at other wavelengths to produce reference grids for various surveys. This requires the maintenance of the accuracy of the Gaia optical reference frame at an appropriate density that is useful for new surveys and is a science objective in itself as it lies at the heart of fundamental astrometry. The Gaia celestial reference frame will immediately supercede any other optical reference frames and will be the standard optical reference frame for the astronomical community for many years. Nevertheless, a new Gaia-like mission will be necessary in the coming decades in order to maintain the optical realization of the celestial reference frame. This on its own is a strong science case but the addition of NIR astrometry will increase the density of this grid in obscured regions and provide a link to the ICRF in a new wavelength region.

\section{Outlook}

The proposal to ESA's call for "New Science Ideas" to develop NIR detectors with TDI mode (Hobbs et al. (2016)) was successful. Since then work has begun to clearly define the science requirements for such a mission and further studies are on-going to determine how best to implement the detector technology.

\section{References}

Hobbs, D. et al. 2016, arXiv - http://adsabs.harvard.edu/abs/2016arXiv160907325H

Friedli, D. et al. 1994, Astrophysical Journal, 430, L105-L108

\&Sellwood, J. A. and Binney, J. J. 2002, Monthly Notices of the RAS, 336, 785-796

Governato, F. et al. 2012, Monthly Notices of the RAS, 422, 1231-1240

Kuhn, D. et al. 2015, Astrophysical Journal, 812, 131

Carrasco, J. M. et al. 2014, Astronomy \& Astrophysics, 565, A11

Gnedin, O. Y. et al. 2005, Astrophysical Journal, 634, 344-350 